\title{
Strange behavior of persistent currents in small Hubbard rings
}

\author{
Santanu K. Maiti, J. Chowdhury and S. N. Karmakar* \\ Saha Institute of Nuclear Physics, 1/AF, Bidhannagar, Kolkata 700 064, India
}

\begin{abstract}
We show exactly that small Hubbard rings exhibit unusual kink-like structures giving anomalous oscillations in persistent current. Singular behavior of persistent current disappears in some cases. In half-filled systems mobility gradually drops to zero with interaction, while it converges to some finite value in nonhalf-filled cases.
\end{abstract}

PACS No.: 73.23.Ra, 71.27.+a, 73.23.-b

Keywords: Persistent Current, Electronic Structure, Hubbard Correlation, and Drude Weight.

\footnotetext{
* Corresponding Author:

Email address: sachin@cmp.saha.ernet.in
} 
Since the pioneering work of Büttiker, Imry, and Landauer [1] the phenomenon of persistent current in mesoscopic systems has been discussed theoretically in various papers 2, , 3, 4, 5, 6, 7, 8, 9, 10, 11, 12, 13. Although this phenomenon is thought to be qualitatively understood within the framework of oneelectron theory 1, 2, 3, 4, 5, 6, it has proved difficult to explain recent experimental results [14, 15, 16, 17. The unexpected large amplitude of the measured currents, the observation of currents with both $h c / e$ and $h c / 2 e$ flux periodicity, and, the sign of the currents are the major subjects of disagreement. There are many theoretical attempts to explain the experimental results, and it is generally believed that disorder 5, 6, 9, 10, 11 and electron-electron correlation [7, 8, 9, 10, 11, 12, 13, are the key factors which can resolve the controversy between theory and experiment. However, no consensus on the role of interaction and disorder has yet been reached. The explanation of the experimental results become even much more illusive since recently Kravtsov et al. [19] have shown that additional currents may be generated in the rings by other mechanisms which are not experimentally distinguishable from persistent current.

To understand the precise role of correlation, in this letter we concentrate on the exact calculation of persistent current and Drude weight in ordered 1D Hubbard rings threaded by magnetic flux. Our study reveals that certain aspects of the many-body effects on persistent current have not been discussed in the literature, and we will see that Hubbard correlation leads to many remarkable effects. We limited ourselves to small Hubbard rings and these studies might be also helpful to understand the physical properties of TTF-TCMQ conductors, various aromatic molecules and systems of connected quantum dots 8 . With the new technological advancements, it is now possible to fabricate such small rings, and, in a recent experiment Keyser et al. 18 reported the evidence of anomalous Aharonov-Bohm oscillations from the transport measurements on small quantum rings with less than ten electrons. In these small rings with few number of electrons, the electron-electron interaction becomes important as the Coulomb potential is not screened much, and we will show that the electronelectron interaction leads to similar anomalous oscil- lations in the persistent current as a function of the magnetic flux.

We use Hubbard model to represent the system, which for a $N$-site ring enclosing a magnetic flux $\phi$ (in units of elementary flux quantum $\phi_{0}=h c / e$ ) can be expressed as

$$
H=t \sum_{i, \sigma}\left[e^{i \Theta} c_{i, \sigma}^{\dagger} c_{i+1, \sigma}+h . c .\right]+U \sum_{i} n_{i \uparrow} n_{i \downarrow}
$$

where $\Theta=2 \pi \phi / N$, and the other symbols have their usual meanings. We take $t=-1$ and use the units $c=1, e=1$.

At zero temperature, the persistent current in the ring threaded by flux $\phi$ is determined by $[3$

$$
I(\phi)=-\frac{\partial E_{0}(\phi)}{\partial \phi},
$$

where $E_{0}(\phi)$ is the ground state energy. We evaluate this quantity exactly to understand unambiguously the role of interaction on persistent current, and this is achieved by exact diagonalization of the many-body Hamiltonian Eq. (11).

We also consider the response of the system to a uniform time-dependent electric field in terms of the Drude weight [20, 21] $D$, a closely related parameter that characterizes the conducting nature of the system as originally noted by Kohn 22. The Drude weight can be calculated from the relation 10

$$
D=\frac{N}{4 \pi^{2}}\left(\frac{\partial^{2} E(\phi)}{\partial \phi^{2}}\right)_{\phi=\phi_{\min }},
$$

where $\phi_{\min }$ is the location of the minimum of $E_{0}(\phi)$. Now we present below the results of our calculations for the various systems.

We first consider two electron systems, and to have a deeper insight to the problem, we begin our discussion with the simplest possible system which can be treated analytically up to certain level. This is the case of a three-site ring consisting of two opposite spin electrons. The Hamiltonian is a $(9 \times 9)$ matrix which can be block diagonalized to $(6 \times 6)$ and $(3 \times 3)$ matrices by proper choice of the basis states. This can be done by constructing basis states for each sub-space with a definite value 
Table 1: Eigenvalues $(\lambda)$ and eigenstates of two opposite spin electrons for $\mathrm{N}=3$.

\begin{tabular}{|c|c|c|c|c|c|c|}
\hline \multirow{2}{*}{$\begin{array}{c}\text { Total } \\
\text { spin } S\end{array}$} & \multicolumn{3}{|r|}{$\bar{U}=0$} & \multicolumn{3}{|c|}{$U=2$} \\
\hline & $\bar{\lambda}$ & Degeneracy & Eigenstate & $\bar{\lambda}$ & Degeneracy & Eigenstate \\
\hline \multirow[b]{3}{*}{0} & -4 & 1 & $\left(-\frac{1}{\sqrt{2}},-\frac{1}{\sqrt{2}},-\frac{1}{\sqrt{2}},-1,-1,1\right)$ & $-2 \sqrt{3}$ & 1 & $(a, a, a,-1,-1,1)$ \\
\hline & -1 & 2 & $\begin{array}{c}(0, \sqrt{2},-\sqrt{2}, 1,0,1) \\
(-\sqrt{2}, 0, \sqrt{2},-1,1,0)\end{array}$ & 0 & 2 & $\begin{array}{l}\left(0, \frac{1}{\sqrt{2}},-\frac{1}{\sqrt{2}}, 1,0,1\right) \\
\left(-\frac{1}{\sqrt{2}}, 0, \frac{1}{\sqrt{2}},-1,1,0\right)\end{array}$ \\
\hline & 2 & 3 & $\begin{array}{c}\left(\frac{1}{\sqrt{2}}, 0, \frac{1}{\sqrt{2}}, 0,0,1\right) \\
\left(0,-\frac{1}{\sqrt{2}},-\frac{1}{\sqrt{2}}, 0,1,0\right) \\
\left(-\frac{1}{\sqrt{2}},-\frac{1}{\sqrt{2}}, 0,1,0,0\right)\end{array}$ & $\begin{array}{c}3 \\
2 \sqrt{3}\end{array}$ & 1 & $\begin{array}{c}(0,-\sqrt{2}, \sqrt{2}, 1,0,1) \\
(\sqrt{2}, 0,-\sqrt{2},-1,1,0) \\
(b, b, b,-1,-1,1)\end{array}$ \\
\hline 1 & $\begin{array}{c}-1 \\
2\end{array}$ & $\begin{array}{l}2 \\
1\end{array}$ & $\begin{array}{c}(1,0,1) \\
(-1,1,0) \\
(-1,-1,1)\end{array}$ & $\begin{array}{c}-1 \\
2\end{array}$ & $\begin{array}{l}2 \\
1\end{array}$ & $\begin{array}{c}(1,0,1) \\
(-1,1,0) \\
(-1,-1,1)\end{array}$ \\
\hline
\end{tabular}

of the total spin $S$. The basis set for the sixdimensional sub-space is chosen as $\mathcal{A} \equiv\{\mid \uparrow \downarrow, 0,0>$, $|0, \uparrow \downarrow, 0>| 0,0,, \uparrow \downarrow>,(|\uparrow, \downarrow, 0>-| \downarrow, \uparrow, 0>) / \sqrt{2}$, $(|0, \uparrow, \downarrow>-| 0, \downarrow, \uparrow>) / \sqrt{2},(|\downarrow, 0, \uparrow>-| \uparrow, 0, \downarrow>) / \sqrt{2}\}$ (each with $S=0$ ), while that for the other subspace is taken as $\mathcal{B} \equiv\{(|\uparrow, \downarrow, 0>+| \downarrow, \uparrow, 0>) / \sqrt{2}$, $(|0, \uparrow, \downarrow>+| 0, \downarrow, \uparrow>) / \sqrt{2},(|\uparrow, 0, \downarrow>+| \downarrow, 0, \uparrow>) / \sqrt{2}\}$ (each with $S=1$ ). For $U=0$ and $U=2$, in Table 1 we list the energy eigenvalues, eigenstates and the degeneracy of the levels of this system in the absence of any magnetic field. In this table we set $a=-\sqrt{2} /(1+\sqrt{3})$ and $b=\sqrt{2} /(-1+\sqrt{3})$. Both for $U=0$ and $U \neq 0$ cases, the two-fold degeneracy of the energy levels corresponds to the $C_{3 v}$ symmetry of the system, and, this degeneracy gets lifted by the magnetic flux. It is apparent from this table that the eigenvalues, eigenstates and also the degeneracy of the levels are not affected by correlation in the threedimensional sub-space (the reason is that basis set $\mathcal{B}$ does not involve any doubly occupied site), but this is not true in the other sub-space.

The insertion of magnetic flux $\phi$ does not alter the above structure of the Hamiltonian, though it becomes now field dependent. In Fig. 1(a) we have plotted some of the low-lying energy levels $E(\phi)$ 's as functions of $\phi$ (say, $E-\phi$ curves) of this three-site ring with $U=0$, and, the corresponding $I(\phi)$ versus $\phi$ curve (say, $I-\phi$ curve) is shown in Fig. I (b). The $E-\phi$ and $I-\phi$ curves for $U=2$ case are respectively given in Fig. 廿(c) and Fig. 廿(d). In Fig. 廿(a)
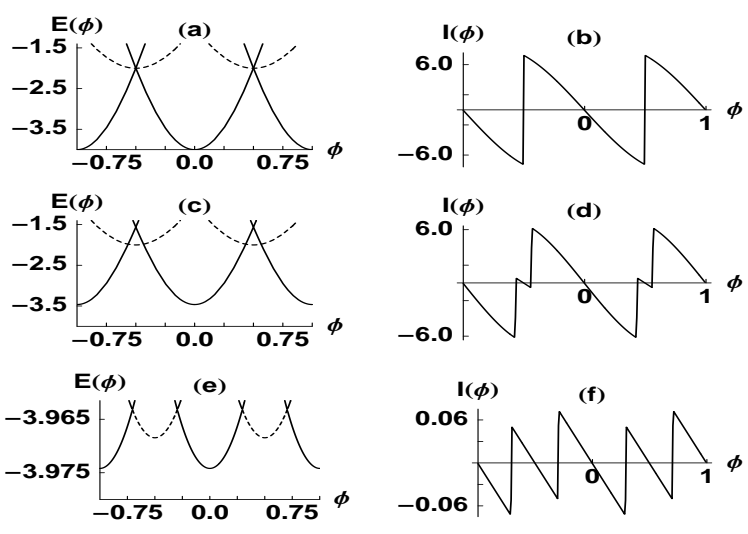

Figure 1: The $E-\phi$ and $I-\phi$ curves for following two $(\uparrow, \downarrow)$ electron systems : i) $N=3, U=0$ in (a) \& (b); ii) $N=3, U=2$ in (c) \& (d); and iii) $N=25$, $U=3$ in (e) \& (f).

and Fig. 1(c), the dotted curves represent the $U$ independent energy levels as mentioned above. Quite interestingly Fig. 1(c) shows that even in the presence of interaction, one of the $U$-independent energy levels becomes the ground state energy level in certain intervals of $\phi$ (e.g., around $\phi= \pm 0.5$ ). In other regions of $\phi$ we have the expected results, i.e., the ground state energy increases due to correlation and the $E_{0}-\phi$ curves become much shallow. As a result, the usual sawtooth shape of the $I-\phi$ characteris- 
tics of $U=0$ case changes drastically as shown in Fig. 1(d), and the role of correlation is quite complex rather than a simple suppression of the persistent current as predicted earlier. A sudden change in the direction and magnitude of persistent current occurs, solely due to correlation, around $\phi= \pm 0.5$, and, it forms kink-like structures in the persistent current as illustrated in Fig. 1(d). The kinks become wider as we increase $U$, and, the most surprising result is that the persistent current remain invariant inside the kinks irrespective of correlation. It is also seen that correlation does not affect the $\phi_{0}$ flux periodicity of the persistent current.

With the above background, we next investigate the role of correlation on persistent current in larger mesoscopic rings. In Fig. 1(e) and Fig.1(f) we display respectively the $E-\phi$ and $I-\phi$ curves for a ring containing two opposite spin electrons with $N=25$ and $U=2$. These figures respectively resembles Fig. 1(c) and Fig.11(d), and it implies that correlation plays the same role in both the cases. Thus we can precisely say that away from half-filling, a ring consisting of two opposite spin electrons always exhibits kinks in the persistent current for any non-zero value of $U$.

Next we consider rings with two up and one down spin electrons as representative examples of three electron systems. In Fig. 2(a) and Fig. 2(b), we respectively plot the $E-\phi$ and $I-\phi$ curves for the half-filled situation ( $N=3$ and $n=3$, where $n$ denotes the number of electrons) with $U=3$. We find that correlation just diminishes the magnitude of the current compared to that of the noninteracting case. In Fig. 2(c) and Fig. 2(d) we respectively plot the $E-\phi$ and $I-\phi$ curves of a non-half-filled situation with $N=10$ and $U=3$, while Fig. 2(e) and Fig. 2(f) are these curves for the $U=15$ case. The behavior of the persistent current, as a function of flux, are quite different at low and high values of $U$. It is evident from Fig. 2(c) that for low $U$, all the $U$-independent energy levels (dotted curves) always lie above the ground state energy of the system, and Fig. 2(d) shows that, apart from a reduction of the persistent current, the $I-\phi$ curve looks very similar to that of a ring without any interaction. On the other hands, we see from Fig. 2(e) that for high $U$, one of the $U$-independent energy levels
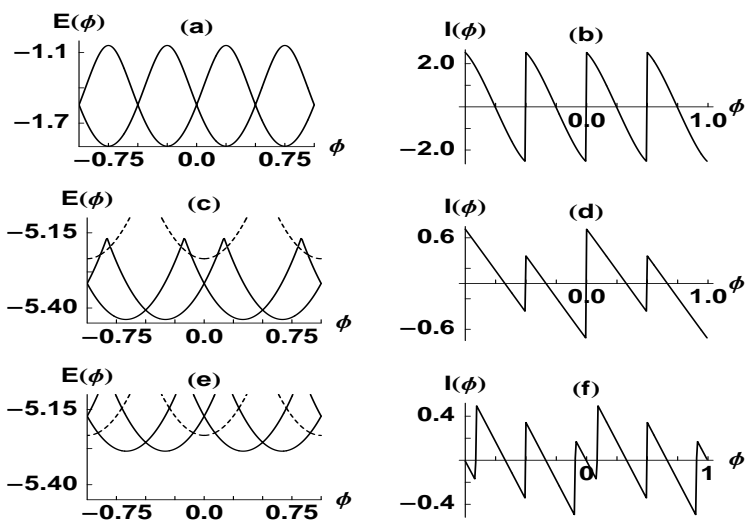

Figure 2: The $E-\phi$ and $I-\phi$ curves for following three $(\uparrow, \uparrow, \downarrow)$ electron systems : i) $N=3, U=3$ in (a) \& (b); ii) $N=10, U=3$ in (c) \& (d); and iii) $N=10, U=15$ in (e) \& (f).

becomes the ground state energy in certain intervals of $\phi$ (e.g., around $\phi=0)$, and, this produces kinks in the $I-\phi$ characteristics as depicted in Fig. 2(f). Certainly there exists a critical value $U_{c}$ of correlation above which kinks appear in the $I-\phi$ curve. We have verified that these features of the energy spectra and persistent current are the characteristics of any non-half-filled ring with two up and one down spin interacting electrons. We also note that the halffilled cases exhibit $\phi_{0} / 2$ periodic currents, while the non-half-filled cases have $\phi_{0}$ periodicity.

Now we consider rings with two up and two down spin electrons as illustrative examples of four electron systems. Let us first study the half-filled case $(N=4$ and $n=4)$. In the absence of any interaction, this system exhibits sharp discontinuity in persistent current at certain values of $\phi$. However, the influence of correlation is quite dramatic and it makes $I(\phi)$ a continuous function of $\phi$ as depicted in Fig. 3 (b). This is a quite fascinating result since correlation drastically changes the analytic behavior of $I(\phi)$, and we will see that this result also holds true for other half-filled systems with even number of electrons. Away from half-filling, we study an interesting typical case of a six-site ring with two up and two down spin electrons. It may be considered as a doubly ionized benzene-like 

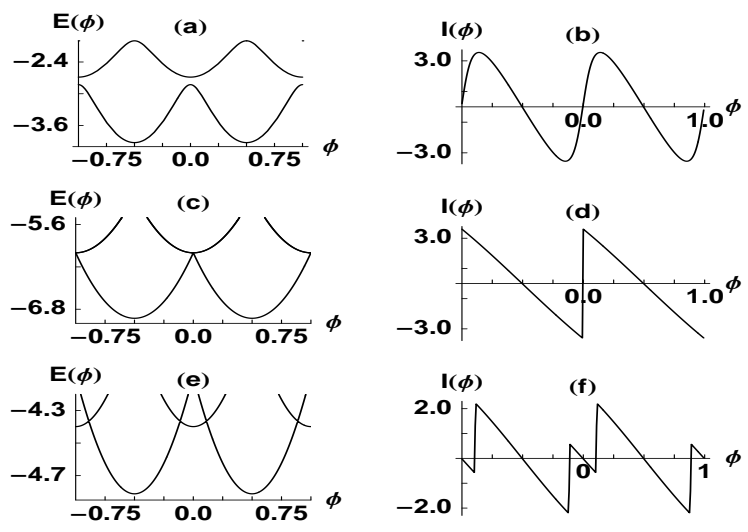

Figure 3: The $E-\phi$ and $I-\phi$ curves for following four ( $\uparrow, \uparrow, \downarrow, \downarrow)$ electron systems : i) $N=4, U=2$ in (a) \& (b); ii) $N=6, U=0$ in (c) \& (d); and iii) $N=6, U=6$ in (e) \& (f).

ring, a system with the promise of experimental verification of our predictions. In Fig. 33 (c) and Fig. 31 (d) we respectively plot the $E-\phi$ and $I-\phi$ curves with $U=0$, while Fig. 3(e) and Fig. 3(f) are these plots with $U=6$. Fig. 3(f) shows that kinks appear in the $I-\phi$ curve (e.g., around $\phi=0$ ) for any non-zero value of $U$, but we interestingly note that the kinks are now due to $U$-dependent eigenstates. Both the half-filled and non-half-filled rings exhibit $\phi_{0}$ periodic currents.

As specific cases of five electron systems, we study rings with three up and two down spin electrons. The $E-\phi$ and $I-\phi$ curves for the half-filled ring $(N=5$ and $n=5$ ) are plotted in Fig. 4(a) and Fig. 4(b) respectively. Interaction just diminishes the magnitude of the current and we find $\phi_{0} / 2$ periodicity. As a non-half-filled five electron case, we take a singly ionized benzene-like ring $(N=6, n=5)$ and calculate persistent current for low and high values of $U$. In Fig. 4(c) and Fig. 4(d) we respectively draw the

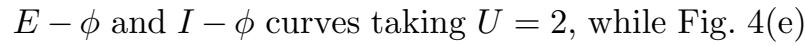
and Fig. 4 (f) are these diagrams with $U=120$. It is clear from Fig. 4(d) and Fig. 目f) that like the three electron cases, kinks appear in the persistent current only after a critical value of $U$. This reveals that the characteristic features of persistent current in mesoscopic Hubbard rings with odd number of electrons
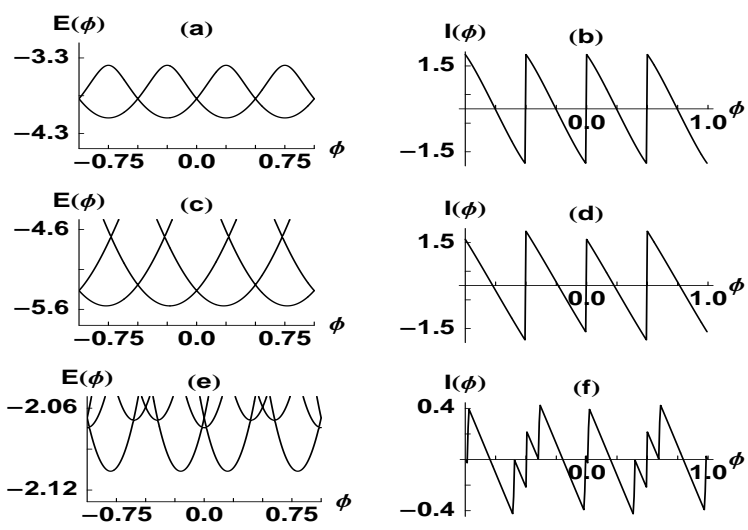

Figure 4: The $E-\phi$ and $I-\phi$ curves for following five $(\uparrow, \uparrow, \uparrow, \downarrow, \downarrow)$ electron systems : i) $N=5, U=2$ in (a) \& (b); ii) $N=6, U=2$ in (c) \& (d); and iii) $N=6, U=120$ in (e) \& (f).

are almost identical.

Lastly, we consider six electron systems, and investigate the nature of persistent current in rings with three up and three down spin electrons. At half-filling (a benzene-like case with $N=6$ and $n=6$ ), we see that $I(\phi)$ becomes a continuous function of $\phi$ as

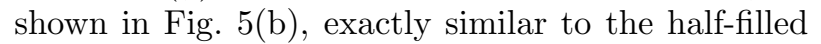
four electron case. Fig. 5 (c) and Fig. 囵(d) are respectively the plot of $E-\phi$ and $I-\phi$ curves for a typical non-half-filled case with $N=7$ and $n=6$, and, we
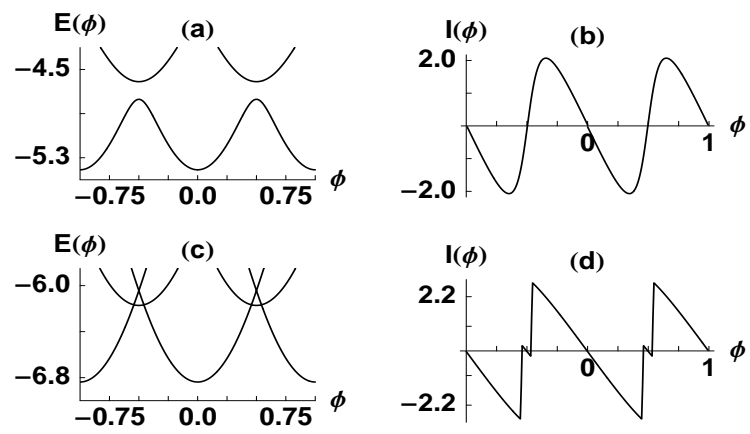

Figure 5: The $E-\phi$ and $I-\phi$ curves for following $\operatorname{six}(\uparrow, \uparrow, \uparrow, \downarrow, \downarrow, \downarrow)$ electron systems : i) $N=6, U=2$ in (a) \& (b) and ii) $N=7, U=2$ in (c) \& (d). 
find striking similarity in the behavior of persistent current with other non-half-filled systems containing even number of electrons. Hence it becomes apparent that mesoscopic Hubbard rings with even number of electrons exhibit similar characteristic features in the persistent current.

In the present context it is also interesting to study the conducting properties of these mesoscopic rings in the presence of Hubbard correlation. For this purpose, we calculate Drude weight $D$ of these systems using Eq. (3). A metallic phase (ballistic regime) will be characterized by a finite value of $D$, while it will be zero in an insulating phase (localized regime) 22 . In Fig. 6(a) and Fig. 6(b), we plot $D$ as a function of
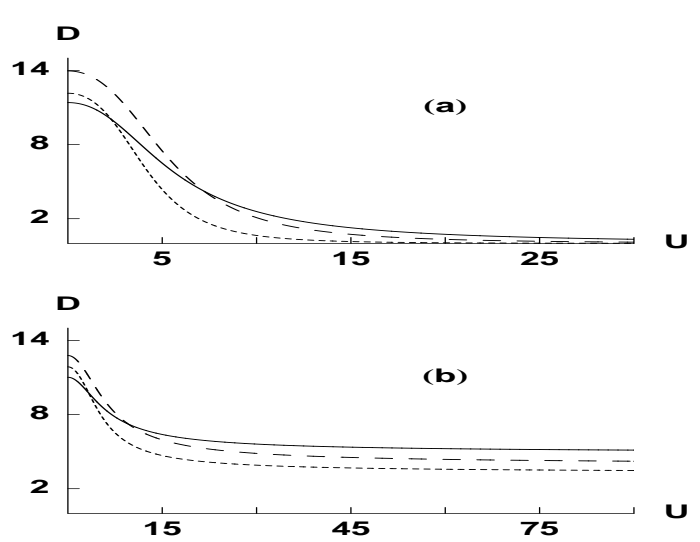

Figure 6: Drude weight vs. interaction for (a) halffilled and (b) non-half-filled systems. The solid, dashed and dotted lines respectively corresponds to 3,4 and 5 electron cases.

$U$ respectively for half-filled and non-half-filled rings with $n=3,4$ and 5 . In the non-half-filled cases, the number of sites corresponding to a given value of $n$ is taken as $N=n+1$, and, we note that other choices of $N$ do not affect the basic features of the $D$ versus $U$ curves. It is evident from Fig. 6(a) that for low $U$, the half-filled systems are in the metallic phase, and, they become insulating only when $U$ is very large. In the insulating phase ground state do not support any empty site, and the situation is somewhat analogous to Mott localization in 1D infinite lattices. Fig. 6 (b) shows that the non-half-filled rings are always con- ducting irrespective of the value of $U$.

In conclusion, we studied exactly the persistent current and Drude weight in small mesoscopic Hubbard rings at zero temperature, and, identified that the Hubbard correlation leads to many interesting new results. The main results are : the appearance of kinks in the persistent current, observation of both $\phi_{0}$ and $\phi_{0} / 2$ periodic currents, no singular behavior of persistent current in the half-filled systems with even number of electrons, evidence of $U$ independent eigenstates, existence of both metallic and insulating phases, etc. We also observe discontinuities in persistent current at non-integer values of $\phi_{0}$ due to electron-electron correlation, which crucially depends on the filling of the ring and also on the parity of the number of electrons. This gives rise to anomalous Aharonov-Bohm oscillations in the persistent current with much reduced period where periodicity is not perfect, and Keyser et al. [18 experimentally observed similar anomalous Aharonov-Bohm oscillations in the conductance of a few-electron quantum ring. Finally, it is worth noting that the above mentioned effects of interaction will be pronounced in strongly correlated electron systems, like, mesoscopic rings of transition metal elements.

We thank R. K. Moitra and B. Bhattacharya for many illuminating discussions.

\section{References}

[1] M. Büttiker, Y. Imry, and R. Landauer, Phys. Lett. 96A, 365 (1983).

[2] R. Landauer and M. Büttiker, Phys. Rev. Lett. 54, 2049 (1985).

[3] H. F. Cheung, Y. Gefen, E. K. Riedel, and W. H. Shih, Phys. Rev. B 37, 6050 (1988).

[4] G. Montambaux, H. Bouchiat, D. Sigeti, and R. Friesner, Phys. Rev. B 42, 7647 (1990).

[5] B. L. Altshuler, Y. Gefen, and Y. Imry, Phys. Rev. Lett. 66, 88 (1991).

[6] F. von Oppen and E. K. Riedel, Phys. Rev. Lett. 66, 84 (1991). 
[7] A. Schmid, Phys. Rev. Lett. 66, 80 (1991).

[8] N. Yu and M. Fowler, Phys. Rev. B 45, 11795 (1992).

[9] M. Abraham and R. Berkovits, Phys. Rev. Lett. 70, 1509 (1993)

[10] G. Bouzerar, D. Poilblanc, and G. Montambaux, Phys. Rev. B 49, 8258 (1994).

[11] T. Giamarchi and B. S. Shastry, Phys. Rev. B 51, 10915 (1995)

[12] D. Weinmann, A. Müller-Groeling, J. -L. Pichard, and K. Frahm, Phys. Rev. Lett. 75, 1598 (1995).

[13] G. Burmeister and K. Maschke, Phys. Rev. B 65, 155333 (2002).

[14] L. P. Levy, G. Dolan, J. Dunsmuir, and H. Bouchiat, Phys. Rev. Lett. 64, 2074 (1990).

[15] D. Mailly, C. Chapelier, and A. Benoit, Phys. Rev. Lett. 70, 2020 (1993).

[16] E. M. Q. Jariwala, P. Mohanty, M. B. Ketchen, and R. A. Webb, Phys. Rev. Lett. 86, 1594 (2001).

[17] R. Deblock, R. Bel, B. Reulet, H. Bouchiat, and D. Mailly, Phys. Rev. Lett. 89, 206803 (2002).

[18] U. F. Keyser, C. Fühner, S. Borck, and R. J. Haug, Phys. Rev. Lett. 90, 196601 (2003).

[19] V. E. Kravtsov and B. L. Altshuler, Phys. Rev. Lett, 84, 3394 (2000).

[20] D. J. Scalapino, R. M. Fye, M. J. Martins, J. Wagner, and W. Hanke, Phys. Rev. B 44, 6909 (1991).

[21] D. J. Scalapino, S. R. White, and S. Zhang, Phys. Rev. B 47, 7995 (1993).

[22] W. Kohn, Phys. Rev. 133, A171 (1964). 